\title{
Valoración del aprendizaje técnico del voleibol mediante la metodología observacional por pares en estudiantes universitarios \\ Assessment of Volleyball Technical Learning Using Peer Observational Methodology in University Students
}

\author{
Javier Álvarez Medina ${ }^{1}$, Víctor Murillo Lorente ${ }^{2 *}$, Jaime Casterad Seral ${ }^{3}$, Alberto Nuviala Nuviala ${ }^{4}$
}

\footnotetext{
${ }^{1}$ Facultad de Ciencias de la Salud y del Deporte de Huesca. Universidad de Zaragoza

2 Facultad de Ciencias de la Salud y del Deporte de Huesca. Universidad de Zaragoza

${ }^{3}$ Facultad de Ciencias de la Salud y del Deporte de Huesca. Universidad de Zaragoza

${ }^{4}$ Facultad de Ciencias del Deporte. Universidad Pablo de Olavide de Sevilla

* Autor para la correspondencia: vmurillo@unizar.es Teléfono: 675569003
}

\section{Resumen}

El Espacio Europeo de Educación Superior defiende metodologías formativas para que los alumnos aprendan a aprender. El objetivo del estudio es conocer la valoración del alumnado sobre el proceso de enseñanza-aprendizaje de la técnica deportiva de voleibol mediante la metodología observacional por pares. Participaron 215 alumnos del Grado de Ciencias de la Actividad Física y el Deporte en la asignatura de segundo curso "Deportes colectivos de colaboraciónoposición" durante los cuatro cursos comprendidos entre 2015 y 2019. Los estudiantes de cada curso se distribuyen en grupos de prácticas diferentes presentando una formación inicial en el ámbito del voleibol diversificada, aunque con todos se realizó la misma intervención en el bloque temático del voleibol durante el primer trimestre. Se ha realizado un estudio de carácter descriptivo en el que se ha calculado la tendencia central y la dispersión. Se ha realizado la comparación de grupos mediante pruebas de MANOVA. Se ha utilizado la escala validada (Álvarez et al., 2020) de evaluación del proceso Enseñanza de la Técnica Deportiva por Pares en la Educación Superior. Los alumnos valoran muy positivamente el proceso de enseñanzaaprendizaje obteniendo medias entre 4.00 y 4.50 sobre 5.00 . La intervención tuvo efectos en la valoración de los alumnos obteniendo diferencias significativas en escala total y factores: diseño, fases, metodología y observación, permitiendo al profesorado mejorar el proceso de enseñanza-aprendizaje de la técnica deportiva del voleibol.

Palabras clave: Metodologías activas, enseñanza por pares, aprendizaje cooperativo, proceso enseñanzaaprendizaje, educación superior.
Cómo citar el artículo:

Álvarez Medina, J., Murillo Lorente, V., Casterad Seral, J., \& Nuviala Nuviala, A. (2022). Valoración del aprendizaje técnico del voleibol mediante la metodología observacional por pares en estudiantes universitarios. Cultura, Ciencia y Deporte, 17 (51), 31-39. http://dx.doi.org/10.12800/ccd.v17i51.1662

\section{Abstract}

The European Higher Education Area defends training methodologies so that students learn to learn. The objective of the study is to know the assessment of the students about the teaching-learning process of the volleyball sports technique through the observational methodology by pairs. 215 students of the Degree in Physical Activity and Sports Sciences participated in the second-year subject "Collaborative-opposition collective sports" during the four years between 2015 and 2019. The students of each course are divided into different practice groups presenting a diversified initial training in the field of volleyball, although the same intervention was carried out with all of them in the thematic block of volleyball during the first trimester. A descriptive study has been carried out in which the central tendency and dispersion have been calculated. The groups were compared using MANOVA tests. The validated scale (Álvarez et al., 2020) has been used to evaluate the process of Teaching Sports Technique by Peers in Higher Education. The students value the teaching-learning process very positively, obtaining averages between 4.00 and 4.50 out of 5.00. The intervention had effects on the evaluation of the students, obtaining significant differences in total scale and factors: design, phases, methodology and observation, allowing teachers to improve the teaching-learning process of volleyball sports technique.

Keywords: Active methodologies, peer teaching, Cooperative learning, Teaching learning process, higher education. 


\section{Introducción}

El Espacio Europeo de Educación Superior (EESS) exige una renovación pedagógica y apuesta por un modelo basado en un tipo de aprendizaje en el que, la metodología fundamental del proceso formativo, permita al estudiante aprender a aprender mediante la adquisición de competencias para el aprendizaje permanente y constructivo (Chiva-Bartoll et al., 2018; Gargallo-López et al., 2020). Esta renovación pedagógica supone un cambio fundamental en el tipo de aprendizaje que deben recibir los alumnos universitarios en la actualidad (Muñoz-San Roque et al., 2016).

Se ha demostrado que el aprendizaje cooperativo entre alumnos es de gran utilidad en la enseñanza de las habilidades técnicas deportivas, siendo posible su aplicación en cualquiera de los diferentes niveles educativos: primaria, secundaria, ciclos formativos, enseñanzas superiores (Gómez \& Gil, 2018; Otero et al., 2014; Salmerón, 2013). El aprendizaje cooperativo se entiende como una estructura de trabajo en la que las decisiones del alumnado están supeditadas a las directrices y condiciones establecidas por el docente (Gómez \& Gil, 2018). Boud (2010) manifiesta que el aprendizaje de los estudiantes es mayor, si junto a los profesores, se convierten en socios responsables del aprendizaje y van asumiendo la responsabilidad de los procesos de enseñanza-aprendizaje (EA) y desarrollando la capacidad para juzgar la calidad de su propio trabajo, así como el de los otros, según unas normas acordadas. Este aprendizaje cooperativo conlleva una reestructuración de la configuración de la enseñanza por modelos de aprendizaje más autónomos y reflexivos en los que cambian las relaciones alumno-profesor y alumnoalumno. Estos modelos de aprendizaje son el punto de inflexión que cambia el foco de la clase de la enseñanza del docente, al aprendizaje del alumnado, donde el profesor facilita el éxito del proceso y el alumno es protagonista directo y activo (Cañadas et al., 2018; del Arco-Bravo et al., 2019; Izquierdo, 2019; Vera-Lacarcel et al., 2008).

La investigación educativa debe ser una actividad científica $y$, por tanto, formal, sistemática, controlada, empírica y objetiva, además debe poseer los atributos del método científico (Tejedor, 2014). Su objetivo básico debe aportar explicaciones razonables de los hechos o fenómenos estudiados, a fin de contribuir a la creación de un cuerpo coherente de conocimientos, orientados a producir la información necesaria para mejorar la acción educativa (Tejedor, 2018). Toda intervención debe ser evaluada de manera objetiva, siendo el cuestionario el instrumento más utilizado para ello (Otero et al., 2014). La mayoría de los cuestionarios deben diseñarse "ad hoc" por las particularidades de cada investigación (León et al., 2017; Villardón-Gallego et al., 2013).

Son diversos los estudios que se centran en estudiar los beneficios y bondades de incorporar, en los procesos de enseñanza-aprendizaje, metodologías participativas y cooperativas. Del Arco et al. (2019), con el objetivo de conocer el efecto del método de enseñanza denominado clase invertida valida un cuestionario formado por cuatro dimensiones: el cambio de rol docente y discente, la integración de las TIC en el proceso de enseñanzaaprendizaje, el desarrollo del proceso de evaluación propuesto y finalmente, el desarrollo de la autonomía. Izquierdo et al. (2019) construye un cuestionario para medir el aprendizaje cooperativo en la formación de maestros de
Educación Primaria. Muñoz-San Roque et al. (2016) validan un cuestionario para medir la autopercepción del nivel de desarrollo de la competencia de aprender a aprender. Gómez y Gil (2018) estudian el estilo de aprendizaje y su relación con la educación entre pares estableciendo cuatro objetivos: analizar las propiedades psicométricas de las puntuaciones del instrumento de medida de las características del compañero tutor; analizar el estilo de aprendizaje predominante del alumnado de la ESO; analizar las respuestas de este alumnado en relación a la educación entre iguales; y contrastar la relación entre el estilo de aprendizaje de este alumnado y sus respuestas relativas al compañero tutor. En definitiva, se analiza la importancia de dar valor al grupo en aras de la promoción de la motivación que contribuya al fomento de los procesos de intercambio de conocimientos entre los estudiantes.

La necesidad de hacer partícipe y protagonista del proceso de enseñanza-aprendizaje al alumnado a través de metodologías activas y participativas, obliga a investigar sobre la evaluación de este estilo de enseñanza, para lo que se construyó y validó un cuestionario ad hoc con el objetivo de evaluar el Proceso de Enseñanza Aprendizaje de la Técnica Deportiva por Pares en la Enseñanza Superior (Etepes) (Álvarez et al., 2020) formado por seis dimensiones: diseño; fases; aprendizaje autónomo; metodología; observación; conocimientos previos.

Así, el objeto de estudio de esta investigación es conocer la valoración del alumnado sobre el proceso de enseñanzaaprendizaje de la técnica deportiva del voleibol, basado en la metodología observacional por pares llevada a cabo en la Educación Superior en el Grado de Ciencias de la Actividad Física y del Deporte que permita mejorar el mismo.

La hipótesis de investigación responde a que los estudiantes participantes en la enseñanza del voleibol mediante la metodología observacional por pares perciben de forma positiva y eficaz el aprendizaje de la técnica deportiva.

\section{Método}

Participantes

Estudio cuasiexperimental longitudinal-transversal con medidas pre y post intervención. Participaron 215 alumnos del Grado de Ciencias de la Actividad Física y el Deporte de la Universidad de Zaragoza, todos matriculados en la asignatura anual de segundo curso "Deportes colectivos de colaboración-oposición" (asignatura obligatoria de 9 créditos) durante los cursos 2015/2016, 2016/2017, 2017/2018 y $2018 / 2019$, impartida en los cuatro años por el mismo profesor el cual es el responsable de la asignatura desde el año 2000.

Los alumnos de cada curso corresponden a un grupo:

- Grupo 1: 2015/2016, 54 alumnos.

- Grupo 2: 2016/2017, 53 alumnos.

- Grupo 3: 2017/2018, 54 alumnos.

- Grupo 4: 2018/2019, 54 alumnos.

El $28.80 \%$ de los estudiantes son de género femenino y el $71.20 \%$ de género masculino. La edad media es de 20 años. 


\section{Instrumento}

Se ha utilizado la escala de evaluación del proceso enseñanza de la técnica deportiva por pares en la educación superior "Etepes" (Álvarez et al., 2020). El instrumento está compuesto por seis dimensiones (Diseño, Fases, Aprendizaje, Metodología, Observación y Conocimientos previos) y 21 ítems, con una fiabilidad medida con alfa de Cronbach de .91 en la prueba inicial y .94 en la prueba final. La escala es de tipo Likert, oscilando entre 1 y $5(1=$ totalmente de acuerdo; $5=$ totalmente en desacuerdo).

Procedimiento

El trabajo de campo se realizó mediante un cuestionario a través de Google Forms con presencia del encuestador antes y al final del programa de intervención. Se solicitó a los participantes que lo cumplimentaran y que consultaran cualquier duda que tuvieran con los ítems. El tiempo invertido en la realización fue de unos 10 minutos cada vez. Los criterios de exclusión fueron:

- No haber realizado alguno de los test (pre-post).

- Haber faltado a más de un $10 \%$ de la intervención educativa.

La universidad de origen de la investigación avaló el proceso a través de su aceptación dentro de los programas de Innovación Docente de la Universidad de Zaragoza.

Durante todo el estudio se garantizó el anonimato de las respuestas en cumplimiento de las normas éticas de investigación del Código de Buenas Prácticas en Investigación de la Universidad de Zaragoza (2018), administrando y siendo aceptado por los participantes el correspondiente consentimiento informado para participar en el estudio.

\section{Intervención}

La intervención se ha realizado a través de la metodología observacional por pares, en el bloque temático del voleibol durante el primer trimestre de cada curso en el que los estudiantes trabajan juntos para mejorar su propio aprendizaje y el de su pareja. Es necesario tener en cuenta que en el proceso de EA de una modalidad como el voleibol, con respuestas inciertas y abiertas, el deportista debe ser capaz de atender primero a los mecanismos de percepción y de decisión para acabar con el mecanismo de ejecución "fundamento técnico-táctico utilizado" (Marteniuk, 1976), eso supone mucho más tiempo de práctica para dominar los tres mecanismos de intervención motora que se dan prácticamente de manera simultánea (Álvarez, 2011). Es clave adaptar constantemente este contexto cambiante para facilitar la mejora global del alumno, facilitando la interpretación del juego y su disfrute. La observación directa e indirecta de todo este proceso y su análisis y corrección posterior ayuda al alumno a asimilar mejor y antes su aprendizaje técnico-táctico y el de su pareja.

La intervención quedó compuesta por 14 sesiones presenciales y dos no presenciales utilizando un modelo pedagógico conocido como "aula invertida" que transfiere el conocimiento teórico fuera del aula y permite utilizar el tiempo de clase para facilitar la adquisición y práctica de conocimientos (del Arco-Bravo et al., 2019). Este modelo pedagógico permite al alumno ser el protagonista de su aprendizaje y a partir de unas orientaciones iniciales del docente sea capaz de aprender de forma autónoma gracias a la observación y el análisis, en este caso de la técnica deportiva del voleibol, siempre bajo la supervisión y guía del profesor responsable.

Las fases de este proceso pedagógico fueron:

1. Formación inicial teórico-práctica.

En las ocho primeras clases, cuatro teóricas y cuatro prácticas, se enseñan los fundamentos técnico-tácticos, así como los errores más comunes bajo las directrices del profesor.

2. Diseño autónomo de la hoja de observación (supervisado por el profesor).

3. Aplicación de la hoja de observación directa y grabación.

En la clase práctica cinco se realiza el análisis de observación directa de los fundamentos técnicotácticos y grabación por parte de los alumnos.

4. Aplicación de la hoja de observación indirecta a través de la grabación.

En el seminario uno los alumnos realizan esta observación en el laboratorio.

5. Diseño autónomo de tareas de corrección (supervisada por el profesor).

6. Aplicación de tareas de corrección.

En la clase práctica seis y siete, los alumnos aplican las tareas de corrección diseñadas por ellos a su pareja.

7. Segunda aplicación de la hoja de observación directa y grabación.

En la clase práctica ocho se realiza el análisis de observación directa de los fundamentos técnicotácticos y grabación por parte de los alumnos.

8. Segunda aplicación de la hoja de observación indirecta a través de la grabación y evaluación del proceso.

En el seminario dos los alumnos realizan esta observación en el laboratorio y valoran el proceso.

Se han determinado cuatro grupos diferentes formados por los alumnos matriculados en segundo curso en la asignatura en los últimos cuatro cursos (grupo 1: 2015/2016; grupo 2: 2016/2017; grupo 3: 2017/2018; grupo 4: 2018/2019). A cada uno se le aplicó un proceso formativo diferente, diferenciándose por la formación previa y las modificaciones realizadas en el proceso. La formación previa corresponde a si en el primer curso han utilizado este tipo de metodología observacional por pares y las modificaciones corresponden al intento de mejora del proceso realizado, teniendo en cuenta los resultados obtenidos en las intervenciones anteriores, ya que la mejor investigación educativa será aquella que contribuya a mejorar la práctica (Tejedor, 2018). Siguiendo a Gessa (2011), un programa se debe juzgar en términos de su efectividad para ayudar al profesorado a maximizar el aprendizaje del alumnado, debe ser un punto de partida para la toma de decisiones, gracias a la retroalimentación obtenida a partir de los resultados, que ayuden a mejorar el proceso. La formación inicial puede tener repercusiones en la toma pre y las modificaciones del proceso en la toma post intervención.

Los grupos quedaron de la siguiente forma:

Grupo 1:

Cultura, Ciencia y Deporte | AÑO 2022 | volumen 17 | NUM. 51 | España | PÁG. 31 A39 | ISSN: 1696-5043 
- Formación previa: los alumnos en primer curso no han recibido formación previa específica sobre la metodología observacional por pares antes de la intervención.

- Modificaciones: no hay al ser el primer grupo que realiza el proceso.

\section{Grupo 2:}

- Formación previa: los alumnos han recibido formación previa en la asignatura de primer curso de "Actividades acuáticas", concretamente en la enseñanza de estilos de natación a través de la metodología observacional por pares. La hoja de observación en esta asignatura se les da hecha.

- Modificaciones: en cuanto al análisis de la técnica deportiva a través de la observación indirecta en laboratorio se sugiere el uso de programas como "Kinovea" de descarga gratuita a través de la red.

\section{Grupo 3:}

- Formación previa: los alumnos han recibido la misma formación previa que el grupo 2 .

- Modificaciones: desde el inicio de curso los alumnos tienen clara la fundamentación teórica, los objetivos y el proceso que se va a llevar a cabo gracias a la lectura de un documento explicativo en formato de artículo científico. Además, en el proceso realizado se le da más importancia a la adecuada construcción de la hoja de observación y a la observación indirecta a través del programa Kinovea explicado en el laboratorio. Por último se les explica a los alumnos las diferencias del aprendizaje en modalidades eminentemente técnicas y sin variaciones en el entorno, como la natación, y en deportes colectivos en cuestiones de mecanismos de ejecución motriz y tiempo necesario para el aprendizaje. El objetivo de esta intervención es que entiendan las fases del proceso y las apliquen siendo conscientes desde el principio de que el tiempo dedicado es insuficiente.

\section{Grupo 4:}

- Formación previa: los alumnos han recibido la misma formación previa que el grupo 2 y 3.

- Modificaciones: ninguna, no se realiza ninguna modificación diferente al grupo 3 .

\section{Análisis de datos}

Se utilizó el paquete estadístico de SPSS versión 22. Se ha realizado un estudio de carácter descriptivo en el que se ha calculado la tendencia central (a través de la media) y la dispersión (con la desviación estándar). Se ha realizado la comparación de grupos mediante pruebas de MANOVA. La $d$ de Cohen se utilizó para calcular los tamaños del efecto. El nivel de significación se estableció en $p<0.05$.

\section{Resultados}

Los resultados de la efectividad de la intervención relativa al proceso enseñanza de la técnica deportiva por pares en estudiantes universitarios se muestran en la tabla 1. El efecto de interacción (Grupo x Tiempo) resultó ser significativo en la escala total (ETEPES) $\left(p<.001\right.$, Eta $\left.^{2}=.108\right)$ y en sus factores Diseño $\left(p<.05, E t a^{2}=.052\right)$, Fases $\left(p<.05, E t a^{2}=.051\right)$, Metodología $\left(p<.05, E_{t a}^{2}=.064\right)$ y Observación $\left(p<.05, E t a^{2}=\right.$. 034). Los resultados del grupo 1 , tanto en el pre-test como en post-test han sido inferiores al resto de grupos. Los grupos 3 y 4 han tenido valoraciones más altas que el resto de grupos. 
Tabla 1. Efectividad de la intervención en el proceso de enseñanza de la técnica deportiva por pares en estudiantes universitarios

\begin{tabular}{|c|c|c|c|c|c|c|}
\hline \multirow{2}{*}{ Constructo } & \multirow{2}{*}{ Grupo } & \multirow{2}{*}{$\begin{array}{l}\text { Pre } \\
\text { Media } \pm S D\end{array}$} & \multirow{2}{*}{$\begin{array}{l}\text { Post } \\
\text { Media } \pm S D\end{array}$} & \multirow{2}{*}{$\begin{array}{l}\text { Cambio durante } \\
\text { la intervencón }\end{array}$} & \multicolumn{2}{|c|}{ Efecto Grupo x tiempo } \\
\hline & & & & & $p$ & $E t a^{2}$ \\
\hline \multirow{4}{*}{ ETEPES } & 1 & $3.89 \pm .66$ & $3.75 \pm .83$ & $\mathbf{v} 0.14$ & \multirow{4}{*}{.000} & \multirow{4}{*}{.108} \\
\hline & 2 & $4.16 \pm .37$ & $3.94 \pm .44$ & $\mathbf{v} 0.22$ & & \\
\hline & 3 & $4.34 \pm .36$ & $4.32 \pm .37$ & $\mathbf{v} 0.02$ & & \\
\hline & 4 & $4.35 \pm .37$ & $4.40 \pm .31$ & $\Delta 0.05$ & & \\
\hline \multirow{4}{*}{ DISEÑO } & 1 & $4.08 \pm .83$ & $3.95 \pm .92$ & $\mathbf{v} 0.13$ & \multirow{4}{*}{.011} & \multirow{4}{*}{.052} \\
\hline & 2 & $4.45 \pm .41$ & $4.18 \pm .49$ & $\mathbf{v} 0.27$ & & \\
\hline & 3 & $4.49 \pm .37$ & $4.47 \pm .37$ & $\boldsymbol{\nabla} 0.02$ & & \\
\hline & 4 & $4.52 \pm .37$ & $4.52 \pm .35$ & $=$ & & \\
\hline \multirow{4}{*}{ FASES } & 1 & $4.04 \pm .87$ & $3.88 \pm .92$ & $\mathbf{\nabla} 0.16$ & \multirow{4}{*}{.011} & \multirow{4}{*}{.051} \\
\hline & 2 & $4.37 \pm .49$ & $4.07 \pm .64$ & $\mathbf{v} 0.30$ & & \\
\hline & 3 & $4.46 \pm .45$ & $4.41 \pm .43$ & $\mathbf{v} 0.05$ & & \\
\hline & 4 & $4.47 \pm .47$ & $4.47 \pm .40$ & $=$ & & \\
\hline \multirow{4}{*}{ APRENDIZAJE } & 1 & $3.74 \pm .96$ & $3.68 \pm 1.01$ & $\mathbf{\nabla} 0.06$ & \multirow{4}{*}{.615} & \multirow{4}{*}{.009} \\
\hline & 2 & $4.11 \pm .59$ & $3.95 \pm .67$ & $\mathbf{v} 0.16$ & & \\
\hline & 3 & $4.35 \pm .57$ & $4.31 \pm .60$ & $\mathbf{v} 0.04$ & & \\
\hline & 4 & $4.40 \pm .59$ & $4.42 \pm .54$ & $\Delta 0.02$ & & \\
\hline \multirow{4}{*}{ METODOLOGIAA } & 1 & $3.95 \pm .81$ & $3.66 \pm 1.02$ & $\mathbf{v} 0.29$ & \multirow{4}{*}{.003} & \multirow{4}{*}{.064} \\
\hline & 2 & $4.16 \pm .57$ & $4.02 \pm .61$ & $\mathbf{v} 0.14$ & & \\
\hline & 3 & $4.30 \pm .49$ & $4.37 \pm .54$ & $\Delta 0.07$ & & \\
\hline & 4 & $4.30 \pm .48$ & $4.46 \pm .47$ & $\mathbf{\Delta} 0.16$ & & \\
\hline \multirow{4}{*}{ OBSERVACION } & 1 & $4.04 \pm .92$ & $3.87 \pm 1.09$ & $\mathbf{\nabla} 0.17$ & \multirow{4}{*}{.034} & \multirow{4}{*}{.040} \\
\hline & 2 & $4.39 \pm .62$ & $4.14 \pm .70$ & $\mathbf{v} 0.25$ & & \\
\hline & 3 & $4.44 \pm .55$ & $4.46 \pm .49$ & $\Delta 0.02$ & & \\
\hline & 4 & $4.47 \pm .55$ & $4.57 \pm .41$ & $\Delta 0.10$ & & \\
\hline \multirow{4}{*}{$\begin{array}{l}\text { CONOCIMIENTOS } \\
\text { PREVIOS }\end{array}$} & 1 & $3.47 \pm .80$ & $3.48 \pm .90$ & $\mathbf{v} 0.01$ & \multirow{4}{*}{.533} & \multirow{4}{*}{.010} \\
\hline & 2 & $3.47 \pm .70$ & $3.28 \pm .75$ & $\mathbf{v} 0.19$ & & \\
\hline & 3 & $3.99 \pm .65$ & $3.88 \pm .77$ & $\mathbf{v} 0.11$ & & \\
\hline & 4 & $3.97 \pm .63$ & $3.97 \pm .77$ & $=$ & & \\
\hline
\end{tabular}

$E t a^{2}=$ tamaño del efecto; $p=$ nivel de significación; Pre = valoración previa $;$ Post = valoración posterior; $S D=$ desviación estándar

\section{Discusión}

Las medias obtenidas van a indicar el grado de satisfacción de los alumnos con el proceso llevado a cabo. La diferencia de opinión inicial entre grupos se puede observar en los valores pre y la final en el post. El cambio de opinión y efecto de la intervención relativa al proceso de EA viene de la diferencia entre el pre y el post indicando el efecto de interacción (Grupo x Tiempo) si se han obtenido diferencias significativas. Así, los resultados obtenidos son:

\section{Constructo Escala total (ETEPES)}

Este constructo mide la intervención realizada de manera global, sin dividir en factores. Las medias obtenidas indican como los alumnos están bastante de acuerdo con el proceso EA utilizado, siendo el grupo 1 el único que tanto en la toma pre como post obtiene un valor menor de 4.00. Este resultado puede ser debido a que el grupo 1 no ha tenido formación previa en este tipo de metodología en el curso anterior por lo que su opinión es posible no tenga los suficientes argumentos para darle una valoración mayor, ya que según la autorregulación del aprendizaje a mayor conocimiento mayor capacidad para valorar (Muñoz-San Roque et al., 2016).

Con respecto a la valoración de medidas pre y post entre grupos todos los valores son superiores a su anterior. La mayor diferencia de opinión inicial se da entre el grupo 1 y 2 $(\Delta 0.27)$ que puede ser debido a que el segundo grupo sí tuvo una formación previa específica sobre este tipo de metodología observacional por pares en la asignatura de primer curso, concretamente en Actividades acuáticas en la enseñanza de los estilos de natación. La mayor diferencia de opinión final se da entre los grupos 2 y $3(\boldsymbol{\nabla} 0.38)$ y de forma general puede ser debida a la mayor formación previa recibida al comienzo de la intervención por los grupos 3 y 4 a los cuales se les explico detalladamente todo el proceso a seguir en la asignatura a través de un documento en formato artículo científico elaborado gracias a los resultados obtenidos con anterioridad, que sin duda les da una formación previa muy superior a los grupos 1 y 2 .

El efecto de interacción (Grupo x Tiempo) resultó ser significativo en ETEPES y con un tamaño del efecto grande ( $p$ $\left.<.001, E t a^{2}=.108\right)$. En los tres primeros grupos el efecto de la intervención ha hecho que los valores post sean inferiores al pre siendo mayor en los grupos 1 y 2 y mucho menor en el 3. El grupo 4 consigue que el resultado post sea superior al pre. La mayor diferencia en la intervención se produce en el grupo 2 ( $\boldsymbol{\nabla} 0.22$ ), que puede ser debida a que a pesar de utilizar el mismo tipo de metodología, su aplicación y resultados es diferente según en qué modalidad se aplica debido a las variaciones o no del entorno. Estas variaciones afectan a los clásicos mecanismos de ejecución motriz que participan en el acto motor, ya establecidos por Marteniuk (1976), como son el mecanismo de percepción, decisión y ejecución. Así, el proceso de EA en una modalidad individual como la natación, donde prácticamente no hay variaciones en el entorno, solo hay que prestar atención al mecanismo de ejecución "la técnica", mientras que en un deporte colectivo, con continuas variaciones del entorno, el deportista debe ser capaz de atender primero a los mecanismos de percepción "lectura de la situación de juego y dónde está el móvil" y de decisión "qué tengo que hacer y qué técnica tengo que utilizar" para acabar con el mecanismo de ejecución "fundamento técnico-táctico utilizado" lo que lo hace mucho más difícil. El proceso de EA de cada modalidad hace que para los deportistas principiantes sea más fácil la mejora de la técnica en modalidades predominantemente técnicas que en cualquier deporte colectivo. A su vez, la curva de aprendizaje 
en etapas iniciales de EA es mayor en las modalidades eminentemente técnicas que en los deportes colectivos que van a necesitar mucho más tiempo de práctica para dominar los tres mecanismos de intervención motora que se dan prácticamente de manera simultánea (Álvarez, 2011). La falta de tiempo para asimilar todo el proceso de aprendizaje en el deporte colectivo podría ser la causa de la diferencia negativa entre la toma pre y post del grupo 2, al compararla el alumnado con su experiencia previa en natación. Estas diferencias ya no se dan en los grupos 3 y 4 lo que puede ser debido a las modificaciones introducidas en la intervención y a la explicación realizada a los alumnos sobre el tiempo insuficiente para el asentamiento del proceso realizado.

La intervención ha hecho cambiar la opinión del alumnado obteniendo peor valoración al final de la misma en los dos primeros grupos, igualándose en el tercero y mejorando en el cuarto. Las posibles causas, más concretas a las ya indicadas, se irán analizando en cada uno de los factores.

Todos los factores se comportan de la misma forma encontrando las mayores diferencias de opinión inicial pre entre grupos 1 y 2 , y la final post entre el 2 y el 3 . El mayor efecto de intervención negativa se da en el grupo 2 que se iguala en el 3 y en el 4 es positiva.

De los seis factores que componen el ETEPES cuatro de ellos han obtenido diferencias significativas en el efecto de interacción (Grupo x Tiempo).

\section{Constructo Diseño}

El primero de los factores, "Diseño", hace alusión a la importancia de realizar una adecuada construcción, tanto de la hoja de observación, como de las tareas de corrección. Las hojas de observación/evaluación pueden ofrecer numerosas ventajas como: conducir a los estudiantes hacia un aprendizaje más directo y efectivo; situar a los alumnos en su aprendizaje (nivel de partida) y hacia donde progresa a medida que va repitiendo según la información recibida por su compañero; facilitar la labor del docente; favorecer la responsabilidad del alumno haciéndoles más autónomos e independientes en su aprendizaje; estimular la participación activa del discente en el proceso de aprendizaje, potenciando unas relaciones interpersonales más positivas; fomentar la autoestima en los alumnos y la confianza en sí mismos. La elaboración de este tipo de materiales curriculares ad hoc se considera como motivo de experimentación que se pueden argumentar y modificar a partir de la práctica y de la experiencia, permitiendo mejorar, en definitiva, los procesos de EA. (Gómez-Gonzalvo et al., 2017). La utilización continua de estos instrumentos propios facilita que el alumno tome conciencia y asuma aspectos fundamentales de los contenidos que está aprendiendo, aumentando así su eficacia (Vernetta et al., 2009). Este factor obtiene las medias más altas, alcanzando en el grupo 1 valores de 4.00 y en el resto cercanos al 4.50 mostrando la gran importancia que le dan los alumnos a la construcción de sus propios instrumentos en el proceso. Después de las primeras clases impartidas por el profesor (formación inicial teórico-práctica) los alumnos deben elaborar sus propias fichas-hojas de observación de los fundamentos de la modalidad deportiva que les permitan detectar los errores más importantes de la técnica y poder diseñar tareas adecuadas de corrección, intentado cumplir con los principios de integración de una buena calidad educativa: adecuación a las características de los alumnos universitarios; integración en el proceso de enseñanza-aprendizaje, es decir, debe tener continuidad durante todas las sesiones de la
Unidad Didáctica; relevancia en cuanto a la ejecución final técnica, focalizando la atención en aquellos aspectos técnicos indicados favoreciendo una observación más rigurosa y detallada de los mismos por parte de los alumnos como futuros docentes y formativa ya que implica que los alumnos no sólo sean ejecutantes, sino también evaluadores y correctores de sus compañeros, permitiéndoles una mayor responsabilidad y comprensión de sus aprendizajes, así como una mayor autonomía. El papel del profesor se resume en supervisar el trabajo y aclarar dudas, existiendo siempre la posibilidad de diálogo con los alumnos. Todos los instrumentos elaborados por los alumnos, hojas de observación, tareas para la corrección de los errores pueden irse modificando a lo largo del proceso, reforzando la puesta en práctica de "aprender haciendo", fomentando el trabajo por pares y pequeños grupos donde se genera un clima de aprendizaje y de puesta en común tanto de los conocimientos como de los resultados inmediatos lo que facilita que tomen conciencia y asuman aspectos fundamentales de los contenidos que está aprendiendo, favoreciendo la interiorización del aprendizaje y aumentando así su eficacia (Méndez-Giménez et al., 2016).

El efecto de interacción (Grupo x Tiempo) resultó ser significativo con un tamaño del efecto mediano $\left(p<.05, E_{t a}{ }^{2}\right.$ $=.052$ ). Los valores pre de los grupos 1 y 2 son superiores a los post, obteniendo la mayor diferencia en el grupo 2 ( $\boldsymbol{\nabla}$ 0.27). En el grupo 3 son muy parecidas e iguales en el 4. El diseño de la hoja de observación en natación se les dio hecha, mientras que en esta asignatura deben diseñarla ellos. En cuanto al diseño tanto de la hoja de observación como de las tareas correctivas es importante indicar que es más complejo en las modalidades colectivas donde hay muchas más variables a tener en cuenta que en una modalidad individual sin variaciones en el entorno como se ha explicado con anterioridad.

\section{Constructo Fases}

El segundo constructo denominado "Fases proceso" recoge ítems donde se desglosa la importancia de las diferentes fases utilizadas en el proceso. Las medias obtenidas por encima de 4.00 muestran la conformidad con la importancia de todas las fases del proceso de EA que sin duda van a aplicar en su futuro profesional. El efecto de interacción (Grupo x Tiempo) resultó ser significativo y con un tamaño del efecto mediano $(p<.05$, $\left.E t a^{2}=.051\right)$. La mayor diferencia intervención se vuelve a dar en el grupo 2 ( $\boldsymbol{\nabla} 0.30)$ siendo probablemente la falta de tiempo para aplicar debidamente todas las fases del proceso en el voleibol la causa de la misma.

\section{Constructo Aprendizaje}

El tercero constructo denominado "Aprendizaje autónomo" contiene ítems donde se valora la importancia de la implicación activa del alumno en el proceso a través del cual se hace responsable de su formación. Las medias obtenidas en el grupo 1 y 2 están cercanas a 4.00 y en el 3 y 4 cercanas al 4.50 ratificando la importancia que le da el alumno al uso de metodologías activas que le impliquen en el proceso haciéndole responsable de su formación El aumento de protagonismo y de autonomía se potencia con el trabajo cooperativo que los alumnos deben realizar, en momentos presenciales y no presenciales (del Arco-Bravo et al., 2019), construyendo, en este caso, sus propios instrumentos de observación-evaluación para la identificación de los errores técnico-tácticos más importantes y la confección y aplicación de las tareas de corrección, lo que sin duda ayuda fomenta y 
desarrolla el aprendizaje autónomo y prepara al alumno para afrontar los retos y cambios hacia el futuro durante toda la vida profesional y personal (Gargallo-López et al., 2020). E efecto de interacción (Grupo x Tiempo) no es significativo en este factor.

\section{Constructo Metodología}

El cuarto constructo denominado "Metodología observacional por pares" comprende ítems donde se valora esta metodología frente a las tradicionales y la importancia de la misma en su formación. El aprendizaje basado en problemas y la metodología observacional por pares son los factores que ejercen una influencia mayor y determinante en el desarrollo de las competencias necesarias para transferir conocimiento e innovar en el puesto de trabajo, mayor que el resto de metodologías (Muñoz-San Roque et al., 2016). Las medias obtenidas todas superiores a 4.00, excepto la del grupo 1 , indican la importancia que le dan a este tipo de metodología con respecto a otras más tradicionales. El efecto de interacción (Grupo x Tiempo) resultó ser significativo y con un tamaño del efecto mediano $\left(p<.05, E t a^{2}=.064\right)$. Los grupos 1 y 2 obtienen valores pre superior al post siendo la mayor diferencia la del grupo 1 ( $\mathbf{\nabla} 0.29)$. Los grupos 3 y 4 obtienen valores post superiores al pre $(\Delta 0.07$ y $\Delta 0.16)$ lo que indica que tras el proceso EA los alumnos le dan una mayor importancia para su formación a la metodología observacional por pares con respecto a otro tipo de metodologías tradicionales donde el alumno reproduce las tareas y consignas del docente. Cuando se trabaja en cooperación con los demás, las personas adquieren estrategias de aprendizaje más eficaces y resuelven problemas con un mayor éxito y el aprendizaje por pares, entre iguales se convierte en un eficaz instrumento de aprendizaje activo, en el que además se considera la diversidad del alumnado para intervenir en los procesos de EA en común (Gómez \& Gil, 2018). Además, al final de cada sesión se realiza un feedback grupal de todo lo acontecido, buscando una mayor asimilación de conceptos, ya que un mayor procesamiento deliberativo en la formación da lugar a tener una mayor convicción en esa nueva actitud, lo que, a su vez, determina que esa actitud permanezca más en el tiempo, resista mejor a ataques posteriores de información contra-actitudinal y prediga más eficazmente el proceso cognitivo y la conducta de las personas (Duran, 2010).

\section{Constructo Observación}

El quinto constructo denominado "Observación" recoge ítems donde se exponen los tipos de observación a realizar. Las medias obtenidas a excepción del post del grupo 1 son superiores a 4.00 y cercanas al 4.50 en el grupo 3 y 4 mostrando la gran importancia que le dan los alumnos a la capacidad de observación, fundamental en esta profesión para aprender a enseñar, y ser capaces de identificar los errores en el campo de la motricidad (ejecución técnica). La mayor diferencia entre grupos post se da entre el 2 y 3 $(\Delta 0.32$ ) y el efecto de interacción (Grupo $x$ Tiempo) resultó ser significativo y con un tamaño del efecto pequeño $(p<.05$,

$\left.E t a^{2}=.034\right)$. Esta diferencia entre grupos podría deberse a que en el grupo 2 el docente sugiere pero no obliga ni explica el uso del programa para análisis de la técnica deportiva Kinovea para la observación indirecta, mientras que en el 3 y 4 el docente incide en la importancia de la observación indirecta en el laboratorio, que permite analizar las veces que sean necesarias la técnica grabada, y en el uso del programa Kinovea, explicando en clase las bases de su uso básico. En estos grupos 3 y 4 la diferencia de opinión tras intervención es positiva a favor del post
( $\Delta 0.02$ y $\Delta 0.10$ ), indicando que los alumnos la consideran más importante al final que al principio con valores finales superiores al 4,5. Para aprender a enseñar hay que desarrollar la capacidad de "Aprender a observar". El alumno observador es un alumno que observando aprende, complementa su saber hacer y se convierte en un alumno más autónomo que destaca la información importante. Hay que formar al alumno educador para que sea capaz de ver las cosas que los ojos no expertos no ven, tener la capacidad de discriminar, diferenciar y responder de acuerdo con el contexto que nos encontremos. El alumno debe adquirir estructuras de conocimiento profundas y elaboradas sobre los contenidos de la enseñanza, los aspectos pedagógicos y psicológicos y las características de sus alumnos que le permitan crear un clima adecuado de aprendizaje. Se le debe formar en el conocimiento del movimiento, capacidad de observación que le permita detectar e identificar los aspectos relevantes del mismo, capacidad de diagnóstico y de intervención para la mejora del proceso de enseñanza-aprendizaje (Álvarez, 2011). Al final del proceso los alumnos deben ser capaces de "Diseñar, desarrollar y evaluar los procesos de enseñanzaaprendizaje relativos a la actividad física y del deporte, con atención a las características individuales y contextuales de las personas". Un aspecto importante en la consecución de resultados positivos reside en el hecho de favorecer en el alumnado la toma de decisiones en la elaboración autónoma de las planillas y otras herramientas de observación. Así mismo, se valora como fundamental la inclusión de pautas consensuadas de guía del proceso, contribuyendo así a hacer más efectivos los procesos de autorregulación. (ÁlvarezValdivia, 2017).

\section{Constructo Conocimientos previos}

El sexto constructo denominado "Conocimientos previos" aglutina ítems que exponen la necesidad de tener conocimientos previos para llevar a cabo esta metodología. Es el único factor que da una media inferior a 4.00 en todos los grupos y tomas pre y post. Los alumnos consideran que los conocimientos previos son necesarios pero no tan importantes como otros factores sin haber efecto de interacción (Grupo x Tiempo) significativo.

El trabajo desarrollado reafirma las conclusiones de los estudios que defienden nuevos planteamientos en un entorno de enseñanza orientada al aprendizaje, eje central de la reforma en la que se encuentra inmerso el sistema universitario español, donde los resultados obtenidos van a aportar una retroalimentación valiosa para ayudar a entender y mejorar el proceso establecido ya que, la opinión de los estudiantes sobre los planes de estudio u otros aspectos organizativos y funcionales es fundamental, por ser uno de sus principales protagonistas y además los principales afectados de su acción pedagógica (Martínez-Otero et al., 2018).

Los resultados del presente trabajo muestran como los estudiantes están de acuerdo con los beneficios que aporta la metodología observacional por pares, confirmando lo establecido por otros autores como una de las formas más efectivas para mejorar el desarrollo de estrategias interpersonales, incrementar la intencionalidad formativa, fomentar el diálogo y la capacidad de realizar juicios con los otros compañeros e incluso docentes para evaluar, mejora los procesos y productos del aprendizaje de las material específicas así como el desarrollo de competencias en general, produciendo todo ello un cambio actitudinal hacia el aprendizaje. Los resultados encontrados suponen un indudable valor formativo que ayuda a formar alumnos 
más autónomos, responsables, críticos (Cañadas et al., 2018; del Arco et al., 2019; Ibarra et al., 2012; Moreno et al., 2014; Prins et al., 2005) y como la sociedad demanda, ciudadanos más democráticos (Chiva, 2019; Izquierdo et al., 2019). Los resultados obtenidos tienen que servir como punto de partida de siguientes intervenciones.

\section{Conclusiones}

- Los alumnos valoran muy positivamente el proceso de enseñanza utilizado para la enseñanza de la técnica deportiva a través de la metodología observacional por pares obteniendo medias entre 4.00 y 4.50 sobre 5.00 .

- Las intervenciones han tenido efectos en la valoración de los alumnos obteniendo diferencias significativas en la escala total y en los factores: diseño, fases, metodología y observación, permitiendo al profesorado mejorar el proceso gracias a la retroalimentación recibida.

\section{Aplicaciones prácticas}

Los resultados animan a dar un paso adelante en el proceso de EA y considerar nuevos planteamientos en el diseño, desarrollo y evaluación del mismo (Rodríguez-Gómez et al., 2018) donde la responsabilidad de la evaluación deje de estar solo en el docente y se distribuya dando participación activa al alumnado (Gargallo-López et al. 2017). La participación de los alumnos en la evaluación ya puede ser una valiosa oportunidad para motivar, mejorar y consolidar el aprendizaje, favoreciendo así el aprendizaje a lo largo de la vida y para la vida (Gargallo-López et al., 2020). Moreno et al. (2014), en este mismo Grado, cede la responsabilidad de la evaluación al alumnado y obtiene que este tipo de intervención incrementó la percepción de apoyo a la autonomía, la motivación intrínseca y el valor y rol concedido a la cesión de responsabilidad en la evaluación. Los participantes describieron este programa como un buen sistema de enseñanza-aprendizaje, novedoso y motivador.

Esta perspectiva más progresista ayudará a formar ciudadanos responsables y críticos, ya que la capacidad para evaluar va a ser una habilidad imprescindible a desarrollar en un futuro empleo, y más aún en el contexto de este trabajo realizado en el Grado de Ciencias de la Actividad Física y del Deporte donde se forma a los futuros profesionales de la Educación Física del Sistema Educativo Español.

\section{Referencias}

1. Álvarez, J. (2011). Los deportes colectivos. Teoría y realidad: Desde la iniciación al rendimiento. Zaragoza, Prensas Universitarias de Zaragoza.

2. Álvarez, J., Murillo, V., Casterad. J., \& Nuviala, A. (2020). Validación de la escala del proceso enseñanza de la técnica deportiva por pares en la educación superior (ETEPES). Retos: Nuevas tendencias en educación física, deporte y recreación, 37, 284-290. Recuperado de: https://recyt.fecyt. es/index.php/retos/article/view/73002/45230

3. Alvarez-Valdivia, I. (2017). Evaluar para contribuir a la autorregulación del aprendizaje. Electronic journal of research in educational psychology, 7(19), 1007-1030. https:/ /doi.org/10.25115/ejrep.v7i19.1362

4. Boud (2010). Assessment 2020: Seven propositions for assessment reform in higher education. Sydney:
Australian Learning and Teaching Council. Recuperado de: https://www.uts.edu.au/sites/default/files/Assessment -2020_propositions_final.pdf

5. Cañadas, L., Castejón, F. J., \& Santos-Pastor, M. L. (2018). Relación entre la participación del alumnado en la evaluación y la calificación en la formación inicial del profesorado de educación física. Revista Cultura, Ciencia y Deporte, 13, 291-300.

6. Chiva, Ò., Capella, C., \& Pallarès, M. (2018). Investigaciónacción sobre un programa de aprendizaje-servicio en la didáctica de la educación física. Revista de Investigación Educativa, 36(1), 277-293.

7. Del Arco-Bravo, I., Flores-Alarcia, O., \& Silva, P. (2019). El desarrollo del modelo flipped classroom en la universidad: Impacto de su implementación desde la voz del estudiantado. Revista de Investigación Educativa, 37(2), 451-469. https://doi.org/10.6018/rie.37.2.327831

8. Duran, D. (2010). Cooperative Interactions in Peer Tutoring: Patterns and Sequences in Paired Writing. Middle Grades Research Journal, 5(1), 47-60. Recuperado de: http://grupsderecerca.uab.cat/grai/sites/grupsderecer ca.uab.cat.grai/files/cooperativeinteractions.pdf

9. Gargallo-López, B., Jiménez-Rodríguez, M. Á., MartínezHervás, N., Giménez-Beut, J. A., \& Pérez-Pérez, C. (2017). Métodos centrados en el aprendizaje, implicación del alumno y percepción del contexto de aprendizaje en estudiantes universitarios. Educacion XX1, 20(2), 161-187. h ttps://doi.org/10.5944/educXX1.15153

10. Gargallo-López, B., Pérez-Pérez, C., Garcia-Garcia, F.J., Giménez-Beut, J.A., \& Portillo- Poblador, N. (2020). La competencia aprender a aprender en la universidad: Propuesta de modelo teórico. Educación XX1, 23(1), 19-44. https://doi.org/10.5944/educXX1.23367

11. Gessa, A. (2011). La coevaluación como metodología complementaria de la evaluación del aprendizaje. Análisis y reflexión en las aulas universitarias. Revista de Educación, 354, 749-764. Recuperado de: http://www.revistaeducacio n.educacion.es/re354/re354_30.pdf

12. Gómez, P., \& Gil, A.J. (2018). El estilo de aprendizaje y su relación con la educación entre pares. Revista de Investigación Educativa, 36(1), 221-237. https://doi.org/10.6 018/rie.36.1.233731

13. Gómez-Gonzalvo, Fernando \& Molina, Pere \& MartínezBaena, Alejandro. (2017). El uso de materiales curriculares en Educación Física: racionalidad y desarrollo profesional del profesorado. VIREF. Revista de Educación Física, 6, 9-28.

14. Ibarra, M.S., Rodríguez, G., \& Gómez, M.A. (2012). La evaluación entre iguales: Beneficios y estrategias para su práctica en la Universidad. Revista de Educación, 359, 206-231. https://doi.org/10.4438/1988-592X-RE-2011-359092

15. Izquierd, T., Asensi, E., Escarbajal, A., \& Rodríguez, J. (2019). El aprendizaje cooperativo en la formación de maestros de Educación Primaria. Revista de Investigación Educativa, 37(2), 543-559. https://doi.org/10.6018/rie.37.2.369731

16. León, B., Mendo-Lázaro, S., Felipe-Castaño, E., Polo, M.I., \& Fajardo-Bullón, F. (2017). Team Potency and Cooperative Learning in the University Setting. Revista de Psicodidáctica, 22(1), 9-15. https://doi.org/10.1387/RevPsicodidact.14213

17. Marteniuk, R.G (1976). Information processing in motor skills. New york: Holt, Rinehart, and Winston.

18. Martínez-Otero, Pérez, V., \& Gaeta, L. (2018). Estudio del discurso educativo en una muestra de docentes 
mexicanos. Revista Iberoamericana de Educación, 76(1), 169-186. Recuperado de: https://rieoei.org/RIE/article/view /2855/3837

19. Méndez-Giménez, A., Fernández-Río, J., Rolim Marques, R. J., \& Calderón, A. (2016). Percepciones de estudiantes de máster en Educación Física acerca de los materiales autoconstruidos. Una mirada desde la teoría construccionista de Papert [Physical education master program student perceptions on self-made materials. A reflection from Papert's constructionist theory]. Educación XX1, 19(1), 179-200. https://doi.org/10.5944/educXX1.1558 3

20. Moreno, J.A., Aracil, A., \& Reina, R. (2014). Assignment of responsibility in evaluation: a strategy adapted to the European Higher Education Area. Educación XX1, 17(1), 183-200. https://doi.org/10.5944/educxx1.17.1.10710

21. Muñoz-San Roque, I., Martín-Alonso, J. F., Prieto-Navarro, L., \& Urosa-Sanz, B. (2016). Autopercepción del nivel de desarrollo de la competencia de aprender a aprender en el contexto universitario: Propuesta de un instrumento de evaluación. Revista de Investigación Educativa, 34(2), 369-383. https://doi.org/10.6018/rie.34.2.235881

22. Otero, F., Calvo, Á., \& González-Jurado, J. (2014). Análisis de la evaluación de los deportes de invasión en Primaria. (Analysis of the assessment of invasion sports in elementary school). Revista Cultura, Ciencia y Deporte, 9(26), 139-153. https://doi.org/10.12800/ccd.v9i26.432

23. Prins, F. J., Sluijsmans, D. M. A., Kirschner, P. A., \& Strijbos, J. W. (2005). Formative peer assessment in a CSCL environment: A case study. Assessment and Evaluation in Higher Education, 30(4), 417-444. https://doi.org/10.1080/0 2602930500099219

24. Rodríguez-Gómez, G., Ibarra-Saiz, M. S., \& CuberoIbáñez, J. (2018). Competencias básicas relacionadas con la evaluación. Un estudio sobre la percepción de los estudiantes universitarios. Educación XX1, 21(1), 181-208. h ttps://doi.org/10.5944/educXX1.20184

25. Salmerón, L. (2013). Actividades que promueven la transferencia de los aprendizajes: Una revisión de la literatura. Revista de Educación, Extraordin, 34-53. https://d oi.org/10.4438/1988-592X-RE-2013-EXT-253

26. Tejedor, F. (2014). Investigación educativa: su desarrollo en el ámbito de la tecnología educativa. En A. García-Valcárcel (Coord.), Investigación y tecnología de la información y comunicación al servicio de la innovación educativa ( $\mathrm{pp}$. 176-208). Salamanca: Ediciones Universidad de Salamanca.

27. Tejedor, F.J. (2018). Investigación educativa: La utilidad como criterio social de calidad. Revista de Investigación Educativa, 36(2), 315-330. https://doi.org/10.6018/rie.36.2.3 26311

28. Vernetta, M., López, J., \& Robles, A. (2009). Evaluación compartida con fichas de observación durante el proceso de aprendizaje de las habilidades gimnásticas. Un estudio experimental. Revista Iberoamericana de Educación, 50(2), 1-7. Recuperado de: https://rieoei.org/historico/expe/2864 Santana.pdf.

29. Vera-Lacarcel, J.A., Moreno-González, R., \& MorenoMurcia., J. A. (2008). Relaciones entre la cesión de responsabilidad en la evaluación y la percepción de igualdad en la enseñanza de la educación física escolar. Revista Cultura, Ciencia y Deporte, 4, 25-31.

30. Villardón-Gallego, L., Yániz, C., Atxurra, C., IIraurgi, I., \& Aguilar, M.C. (2013). La competencia para aprender en la universidad: Desarrollo y validación de un instrumento de

Cultura, Cienciay yeporte | AÑo 2022 | volumen 17 | NUM. 51 | España | PÁG. 31 A39 | ISSN: 1696-5043 medida. Revista de Psicodidáctica, 18(2), 357-374. https://do i.org/10.1387/RevPsicodidact.6470

\section{Agradecimientos}

A los programa anuales de Innovación Docente de la Universidad de Zaragoza que aceptaron los siguiente proyectos: Curso 2014-2015 referencia PIIDUZ_14_405: "Valoración de la utilización de la misma metodología docente en las prácticas de deportes del Grado de CCAFD"; 2015-2016, PIIDUZ_15_308: "Uso de la misma metodología observacional por pares en el proceso de enseñanza-aprendizaje de la técnica deportiva en diferentes asignaturas del Grado en Ciencias de la Actividad Física y del Deporte"; 2017-2018, PIIDUZ_17_368: "La evaluación como medio de aprendizaje en la enseñanza de los deportes en la Educación Superior". 